\title{
Phage typing or CRISPR typing for epidemiological surveillance of Salmonella Typhimurium?
}

\author{
Manal Mohammed
}

\begin{abstract}
Objective: Salmonella Typhimurium is the most dominant Salmonella serovar around the world. It is associated with foodborne gastroenteritis outbreaks but has recently been associated with invasive illness and deaths. Characterization of $\mathrm{S}$. Typhimurium is therefore very crucial for epidemiological surveillance. Phage typing has been used for decades for subtyping of S. Typhimurium to determine the epidemiological relation among isolates. Recent studies however have suggested that high throughput clustered regular interspaced short palindromic repeats (CRISPR) typing has the potential to replace phage typing. This study aimed to determine the efficacy of high-throughput CRISPR typing over conventional phage typing in epidemiological surveillance and outbreak investigation of S. Typhimurium.

Results: In silico analysis of whole genome sequences (WGS) of well-documented phage types of S. Typhimurium reveals the presence of different CRISPR type among strains belong to the same phage type. Furthermore, different phage types of S. Typhimurium share identical CRISPR type. Interestingly, identical spacers were detected among outbreak and non-outbreak associated DT8 strains of S. Typhimurium. Therefore, CRISPR typing is not useful for the epidemiological surveillance and outbreak investigation of $S$. Typhimurium and phage typing, until it is replaced by WGS, is still the gold standard method for epidemiological surveillance of $S$. Typhimurium.
\end{abstract}

Keywords: Salmonella Typhimurium, CRISPR typing, Phage typing, Surveillance, Outbreaks

\section{Introduction}

Salmonellosis is one of the most common causes of foodborne disease worldwide. Nontyphoidal salmonellosis (NTS) is a zoonotic disease transmitted from animals to humans through consumption of contaminated food. Worldwide, Salmonella enterica serovar Typhimurium (S. Typhimurium) accounts for most human infection of NTS and has been associated with foodborne outbreaks in developing and developed countries resulting in high morbidity and mortality [1]. Furthermore, the recent emergence of the multidrug-resistant (MDR) S. Typhimurium variant of a distinct Sequence Type ST313 in sub-Saharan Africa represents a major public health concern as it is associated with invasive illness and deaths [2]. An efficient laboratory system for epidemiological

*Correspondence: m.mohammed1@westminster.ac.uk Department of Biomedical Sciences, Faculty of Science and Technology, University of Westminster, London, UK surveillance and outbreak investigation of Salmonella Typhimurium is therefore very crucial.

Phage typing system is a phenotypical method that has been used for decades for subtyping of $S$. Typhimurium to determine the epidemiological relation among isolates [3]. Phage typing is a rapid and low cost approach for the epidemiological surveillance and outbreak investigation of $S$. Typhimurium. The system distinguishes more than 300 definitive phage types (DT) of $S$. Typhimurium based on their patterns of lysis to a unique collection of Salmonella phages but it has shown some limitations including the maintenance of typing phages by the reference laboratory and the updating of the system furthermore it depends entirely on the experience of the individual laboratory for interpretation of the results [4].

Recent studies have suggested that high throughput clustered regular interspaced short palindromic repeats (CRISPRs) typing and the microbead-based CRISPOL assay have the potential to replace traditional bacterial 
typing and subtyping systems including phage typing [5, 6]. CRISPRs consist of direct repeats (DRs) separated by variable spacer sequences that are derived from foreign phages or plasmids [7] while CRISPOL is a bead-based liquid hybridization assay for CRISPR polymorphism [5].

A recent study reported identical CRISPRs between two different phage types of $S$. Typhimurium; DT8 and DT30 [8] which reveals the limitations of CRISPR typing for epidemiological surveillance of $S$. Typhimurium.

This study aimed to analyze the CRISPR/CRISPOL type of well-documented phage types of $S$. Typhimurium in order to determine the efficacy of high-throughput CRISPR and CRISPOL typing over conventional phage typing in epidemiological surveillance of $S$. Typhimurium.

\section{Main text \\ Methods \\ Whole genome sequence of different phage types of $S$. Typhimurium}

The whole genome sequence of well-documented phage types of $S$. Typhimurium (Tables 1,2) were obtained from Enterobase (https://enterobase.warwick.ac.uk/). Furthermore, a set of different phage types of $S$. Typhimurium that are used as control in Anderson phage typing scheme (Tables 1,2) were selected for whole genome sequencing (WGS). Genomic DNA was extracted using QIAamp DNA Mini Kit (Qiagen) according to manufacturer's instructions and submitted for WGS using an Illumina MiSeq on 250 bp paired-end (PE) libraries. The quality of PE data was evaluated using FastQC toolkit (http://www.bioinformatics.babraham.ac.uk/projects/ fastqc/). Adapter sequences were removed using ea-utils package (https://expressionanalysis.github.io/ea-utils/).
PE reads for each isolate were de novo assembled using velvet [9]. The best assembly with the highest N50 value was obtained. Raw sequence data of control phage types of $S$. Typhimurium have been submitted to the European Nucleotide Archive (ENA) under study Accession No.: PRJEB18673 (http://www.ebi.ac.uk/ena/data/view/ PRJEB18673) and also available via Enterobase (https:// enterobase.warwick.ac.uk/).

\section{In silico CRISPR and CRISPOL analysis}

$P E$ reads of different phage types of $S$. Typhimurium were also assembled using Enterobase (https://enterobase.warwick.ac.uk/) where CRISPRs and CRISPOL were called directly from the raw reads rather than the assembly.

Enterobase was used to determine the CRISPR type and CRISPOL type of all phage types of $S$. Typhimurium. In Enterobase, each phage type of $S$. Typhimurium was assigned unique accession number (Tables 1, 2).

Previously, sequenced CRISPR loci of different phage types of $S$. Typhimurium using polymerase chain reaction (PCR) [5] were also included in this study (Table 3).

$\mathrm{PE}$ reads of $S$. Typhimurium phage type DT8 associated with a foodborne outbreak in the summer of 2013 in the States of Jersey [10] were downloaded from ENA; study Accession Number PRJNA248792 (http://www.ebi.ac.uk/ ena/data/view/PRJNA248792) and assembled by Enterobase. CRISPR and CRISPOL types were determined for all outbreak strains using Enterobase (Additional file 1: Table S1).

Spacers sequence within the assembled genomes of outbreak and non-outbreak associated DT8 strains were also characterized using CRISPRFinder (http://crispr. i2bc.paris-saclay.fr/Server/) (Additional file 1: Table S1).

Table 1 Salmonella Typhimurium strains belonging to the same phage type show different CRISPR/CRISPOL type

\begin{tabular}{|c|c|c|c|c|c|c|}
\hline Phage type & Isolate ID (source) & Lab & Accession Number & CRISPR type & CRISPOL type & References \\
\hline \multirow[t]{3}{*}{ DT1 } & DT1 (Clinical isolate) & $\begin{array}{l}\text { Wellcome Trust } \\
\text { Sanger Institute }\end{array}$ & aTraces-0ajzxba (ERS007598) & 8579 & 430 & {$[2]$} \\
\hline & $\begin{array}{l}\text { TM 68-619 (Clinical } \\
\text { isolate) }\end{array}$ & Institut Pasteur & Traces-OMviFiU & 2536 & 54 & Enterobase \\
\hline & $\begin{array}{l}\text { TM 65-111 (Clinical } \\
\text { isolate) }\end{array}$ & Institut Pasteur & Traces-0BvXZSr & 7387 & 90 & Enterobase \\
\hline \multirow[t]{2}{*}{ DT10 } & MS34 (Control DT10) & NSSLRL & bTraces-OeeFHtx (PRJEB18673) & 9509 & 1629 & This study \\
\hline & S81-784 (Clinical isolate) & Institut Pasteur & Traces-0bXCHix & 9913 & 1688 & Enterobase \\
\hline \multirow[t]{2}{*}{ DT15a } & MS41 (Control DT15a) & NSSLRL & bTraces-0FVsVub (PRJEB18673) & 9517 & 1634 & This study \\
\hline & S81-798 (Clinical isolate) & Institut Pasteur & Traces-0QWCSHz & 9916 & 1756 & Enterobase \\
\hline \multirow[t]{3}{*}{ DT41 } & M11-2004 (Control DT41) & NSSLRL & bTraces-OhioJez (PRJEB18673) & 9513 & 1630 & This study \\
\hline & CQ 41 (Clinical isolate) & Institut Pasteur & Traces-0BkvapO & 7434 & 223 & Enterobase \\
\hline & S02-0321 (Clinical isolate) & Institut Pasteur & Traces-0JWTeTs & 9929 & 1766 & Enterobase \\
\hline
\end{tabular}

\footnotetext{
${ }^{a}$ Accession Numbers in Enterobase of clinical isolates of $S$. Typhimurium used in this study. The Accession Number in ENA for each isolate is also provided
}

b Accession Numbers in Enterobase of control phage types of S. Typhimurium sequenced in this study. The Accession Number in ENA is also provided 
Table 2 Salmonella Typhimurium strains belonging to different phage types show identical CRISPR/CRISPOL type

\begin{tabular}{|c|c|c|c|c|c|c|}
\hline Phage type & Isolate ID (source) & Lab & Accession Number & CRISPR type & CRISPOL type & Reference \\
\hline \multicolumn{7}{|c|}{ CRISPR/CRISPOL type among phage types DT8 and DT30 of S. Typhimurium } \\
\hline DT8 & M18-2003 (Control DT8) & NSSLRL & aTraces-0jdDfGp (PRJEB18673) & 1069 & 6 & This study \\
\hline DT8 & DT8 (Clinical isolate) & $\begin{array}{l}\text { Wellcome Trust } \\
\text { Sanger Institute }\end{array}$ & 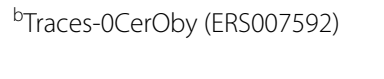 & 1069 & 6 & [2] \\
\hline DT8 & S81-848 (Veterinary isolate) & Institut Pasteur & Traces-OPArkjM & 1069 & 6 & Enterobase \\
\hline DT8 & MS150057 (Clinical isolate) & NSSLRL & Traces-0xVpmwl & 2260 & 708 & Enterobase \\
\hline DT30 & MS57 (Control DT30) & NSSLRL & aTraces-0aYyWix (ERS640854) & 812 & 250 & {$[8]$} \\
\hline DT8 & M12-2001 (Control DT8) & NSSLRL & aTraces-0Jyulvx (PRJEB18673) & 812 & 250 & This study \\
\hline DT8 & M15-2006 (Control DT8) & NSSLRL & aTraces-OWxCKWi (PRJEB18673) & 812 & 250 & This study \\
\hline DT8 & MS32 (Control DT8) & NSSLRL & aTraces-0dPdGho (PRJEB18673) & 812 & 250 & This study \\
\hline \multicolumn{7}{|c|}{ CRISPR/CRISPOL type among phage types DT104, DT104b and U302 of S. Typhimurium } \\
\hline DT104b & MS130531 (Control DT104b) & NSSLRL & 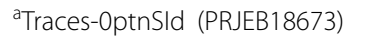 & 12 & 21 & This study \\
\hline U302 & M18-2006 (Control U302) & NSSLRL & aTraces-0rRUdtU (PRJEB18673) & 12 & 21 & This study \\
\hline DT104 & TM75-339 (No data) & Institut Pasteur & Traces-OdpLsNp & 12 & 21 & Enterobase \\
\hline DT104 & MS150098 (Clinical isolate) & NSSLRL & Traces-OVNfjhC & 12 & 21 & Enterobase \\
\hline DT104 & MS150095 (Clinical isolate) & NSSLRL & Traces-0ohRXMQ & 12 & 21 & Enterobase \\
\hline DT104b & MS150159 (Clinical isolate) & NSSLRL & Traces-OmdEaBO & 12 & 21 & Enterobase \\
\hline DT104b & MS150253 (Clinical isolate) & NSSLRL & Traces-OVfHkWp & 7556 & 315 & Enterobase \\
\hline DT104 & MS150005 (Clinical isolate) & NSSLRL & Traces-0ehJIGG & 5000 & 168 & Enterobase \\
\hline \multicolumn{7}{|c|}{ CRISPR/CRISPOL type among phage types DT99, DT56, U319 and DT40 of S. Typhimurium } \\
\hline DT99 & DT99 (Clinical isolate) & $\begin{array}{l}\text { Wellcome Trust } \\
\text { Sanger Institute }\end{array}$ & 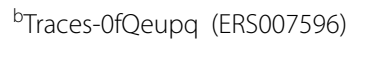 & 7433 & 14 & [2] \\
\hline DT56 & DT56 (Clinical isolate) & $\begin{array}{l}\text { Wellcome Trust } \\
\text { Sanger Institute }\end{array}$ & b'Traces-OWirVGQ (ERS007588) & 7433 & 14 & [2] \\
\hline U319 & U319 (Clinical isolate) & $\begin{array}{l}\text { Wellcome Trust } \\
\text { Sanger Institute }\end{array}$ & 'bTraces-OnXusuL (ERS007613) & 7433 & 14 & [2] \\
\hline DT40 & S05-2864 (Clinical isolate) & Institut Pasteur & Traces-OPxGcXB & 7433 & 14 & Enterobase \\
\hline DT40 & M20-2006 (Control isolate) & NSSLRL & aTraces-OrGCwUc (PRJEB18673) & 9520 & 1637 & This study \\
\hline DT40 & M19-2003 (Control isolate) & NSSLRL & aTraces-0nxmoMB (PRJEB18673) & 9519 & 1636 & This study \\
\hline DT40 & CQ 40 & Institut Pasteur & Traces-OLSHwEV & 745 & 18 & Enterobase \\
\hline \multicolumn{7}{|c|}{ CRISPR/CRISPOL type among phage types DT120, DT7a, DT193 and untypable strains of S. Typhimurium } \\
\hline DT120 & S02-3776 (Clinical isolate) & Institut Pasteur & Traces-OyQDdIW & 9921 & 1759 & Enterobase \\
\hline DT120 & 07_2198 (No Data) & Institut Pasteur & Traces-0pKTfCi & 9911 & 1753 & Enterobase \\
\hline DT120 & M16-2000 (Control DT120) & NSSLRL & aTraces-OfEcWgz (PRJEB18673) & 9510 & 1428 & Enterobase \\
\hline DT7a & MS120840 (Control DT7a) & NSSLRL & aTraces-0psYyDm (PRJEB18673) & 9510 & 1428 & Enterobase \\
\hline DT120 & S/20160374 (Clinical isolate) & SSSCDRL & Traces-OCeRVgg & 322 & 1 & Enterobase \\
\hline DT120 & S/20160407 (Clinical isolate) & SSSCDRL & Traces-OagMeAc & 322 & 1 & Enterobase \\
\hline DT20a & MS150110 (Clinical isolate) & NSSLRL & Traces-0isgxxB & 322 & 1 & Enterobase \\
\hline Untypable & MS150097 (Clinical isolate) & NSSLRL & Traces-OVSIllab & 322 & 1 & Enterobase \\
\hline DT193 & MS150007 (Clinical isolate) & NSSLRL & Traces-OvpTylh & 322 & 1 & Enterobase \\
\hline DT193 & MS150252 (Clinical isolate) & NSSLRL & Traces-OWAKQQZ & 317 & 2 & Enterobase \\
\hline \multicolumn{7}{|c|}{ CRISPR/CRISPOL type among phage types DT12, DT3 and DT193a of S. Typhimurium } \\
\hline DT12 & DT12 (Clinical isolate) & $\begin{array}{l}\text { Wellcome Trust } \\
\text { Sanger Institute }\end{array}$ & '⿳亠口冋丁races-OkmZJki (ERS007564) & 5268 & 19 & {$[2]$} \\
\hline DT12 & S02-2651 (Clinical isolate) & Institut Pasteur & Traces-OFbQprS & 774 & 46 & Enterobase \\
\hline DT3 & S81-482 (Clinical isolate) & Institut Pasteur & Traces-0pUCktc & 5268 & 19 & Enterobase \\
\hline DT3 & S81-531 (Veterinary isolate) & Institut Pasteur & Traces-0pGWuNa & 539 & 13 & Enterobase \\
\hline DT193a & MS120454 (Clinical isolate) & NSSLRL & Traces-OhfCzzz & 774 & 46 & Enterobase \\
\hline \multicolumn{7}{|c|}{ CRISPR/CRISPOL type among phage types DT135, DT191a and RDNC strains of S. Typhimurium } \\
\hline DT135 & DT135 (Clinical isolate) & $\begin{array}{l}\text { Wellcome Trust } \\
\text { Sanger Institute }\end{array}$ & $\begin{array}{l}\text { 'Traces-0xEkcLV } \\
\text { ERS007567 }\end{array}$ & 5753 & 396 & [2] \\
\hline
\end{tabular}


Table 2 continued

\begin{tabular}{|c|c|c|c|c|c|c|}
\hline Phage type & Isolate ID (source) & Lab & Accession Number & CRISPR type & CRISPOL type & Reference \\
\hline DT135 & MS150100 (Clinical isolate) & NSSLRL & Traces-OfqzVBN & 3247 & 66 & Enterobase \\
\hline DT135 & MS150112 (Clinical isolate) & NSSLRL & Traces-0TpmttL & 91 & 4 & Enterobase \\
\hline DT135 & MS150180 (Clinical isolate) & NSSLRL & Traces-0FksMUv & 91 & 4 & Enterobase \\
\hline DT191a & DT191a (Clinical isolate) & $\begin{array}{l}\text { Wellcome Trust } \\
\text { Sanger Institute }\end{array}$ & $\begin{array}{l}{ }^{\mathrm{b}} \text { Traces-0KhAoGt } \\
\text { ERS007574 }\end{array}$ & 91 & 4 & {$[2]$} \\
\hline RDNC & MS150102 (Clinical isolate) & NSSLRL & Traces-0bmnIRV & 91 & 4 & Enterobase \\
\hline RDNC & MS150230 (Clinical isolate) & NSSLRL & Traces-0vTHNcg & 91 & 4 & Enterobase \\
\hline RDNC & MS150009 (Clinical isolate) & NSSLRL & Traces-0Zipaoz & 9404 & 1614 & Enterobase \\
\hline
\end{tabular}

a Accession Numbers in Enterobase of control phage types of $S$. Typhimurium sequenced in this study. The Accession Number in ENA is also provided

b Accession Numbers in Enterobase of clinical isolates of $S$. Typhimurium used in this study. The Accession Number in ENA is also provided

\section{Results}

In silico analysis of genome sequences of control and well documented phage types of $S$. Typhimurium revealed two CRISPR loci, CRISPR-1 and CRISPR-2, within all phage types of $S$. Typhimurium. Although DRs are almost identical among all phage types of $S$. Typhimurium spacers sequences within the CRISPR loci are not unique to the phage type as strains belong to the same phage type have different spacers and subsequently different CRISPR/ CRISPOL type (Table 1) furthermore, different phage types have identical spacers and same CRISPR/CRISPOL type (Table 2).

\section{Different CRISPR/CRISPOL type within the same phage type of $S$. Typhimurium}

In Table 1, three strains of $S$. Typhimurium that belong to phage type DT1 including strains DT1, TM 68-619 and TM 65-111 have different spacers and subsequently show different CRISPR/CRISPOL type; 8579/430, $2536 / 54$ and $7387 / 90$ respectively. Two strains belong to phage type DT10 have different CRISPR/CRISPOL type; MS34 (9509/1629) and S81-784 (9913/1688). Two strains belong to phage type DT15a have different CRISPR/CRISPOL type; 9517/1634 in isolate MS41 and 9916/1756 in isolate S81-798. Moreover, three strains belong to DT41 have different CRISPR/CRISPOL type; $9513 / 1630$ in isolate M11-2004, 7434/223 in isolate CQ 41 and 9929/1766 in isolate S02-0321.

\section{Identical CRISPR/CRISPOL type within different phage types of $S$. Typhimurium CRISPR/CRISPOL type among phage types DT8 and DT30} Identical spacers were detected among different phage types of $S$. Typhimurium. For example, three strains of DT8 including M12-2001, M15-2006 and MS32 have the same CRISPR/CRISPOL type $(812 / 250)$ as a strain belongs to phage type DT30 (MS57). Moreover, different strains belong to phage type DT8 have different CRISPR/CRISPOL type; M18-2003 (1069/6) and MS150057 (2260/708) (Table 2).
Interestingly, $S$. Typhimurium DT8 strains associated with the foodborne outbreak in the summer of 2013 in the States of Jersey [10] showed identical CRISPR/ CRISPOL type (1069/6) however, the same CRISPR/ CRISPOL type were reported in other DT8 strains that do not belong to the outbreak as confirmed by WGS [10]. Identical spacers were detected among outbreak associated and non-outbreak associated DT8 strains (Additional file 1: Table S1).

\section{CRISPR/CRISPOL type among phage types DT104, DT104b and $U 302$}

Variations in the CRISPR/CRISPOL type among strains of the same phage type such as DT104 and DT104b have been also noticed (Table 2). Although three strains of $S$. Typhimurium phage type DT104 including TM75-339, MS150098 and MS150095, have identical spacer sequences and CRISPR/CRISPOL type (12/21) the same CRISPR/ CRISPOL type is present in different phage types including U302 (M18-2006; 12/21) and DT104b (MS130531; 12/21).

\section{CRISPR/CRISPOL type among phage types DT40, DT56, DT99 and U319}

Strains of $S$. Typhimurium belong to different phage types such as DT99, DT56, U319 and DT40 (S05-2864) have identical spacer sequences and identical CRISPR/ CRISPOL type (7433/14). Moreover, several strains belong to phage type DT40 including S05-2864, M202006, M19-2003 and CQ 40 have different CRISPR/ CRISPOL type; 7433/14, 9520/1637, 9519/1636 and $745 / 18$ respectively (Table 2 ).

\section{CRISPR/CRISPOL type among phage types DT7a, DT20a, DT120, DT193 and untypable strains}

In Table 2, strains of $S$. Typhimurium belong to phage type DT120 have different spacers and subsequently different CRISPR/CRISPOL type including S02-3776 (9921/1759), 07_2198 (9911/1753), M16-2000 (9510/1428), and S/20160374 (322/1). 
Table 3 CRISPOL type among different phage types of $S$. Typhimurium

\begin{tabular}{|c|c|c|c|c|c|}
\hline \multirow{2}{*}{$\begin{array}{l}\text { Phage type } \\
\text { DT104 }\end{array}$} & \multirow{2}{*}{$\begin{array}{l}\text { Isolate ID (source) } \\
\text { 02-1540 (Clinical isolate) }\end{array}$} & \multirow{2}{*}{$\begin{array}{l}\text { Lab } \\
\text { Institut Pasteur }\end{array}$} & \multicolumn{2}{|c|}{$\begin{array}{l}\text { Accession number } \\
\text { CRISPR1 locus CRISPR2 locus }\end{array}$} & \multirow{2}{*}{$\begin{array}{l}{ }^{*} \text { CRISPOL type } \\
30\end{array}$} \\
\hline & & & JF724217 & JF724959 & \\
\hline DT104 & 05-2975 (Clinical isolate) & Institut Pasteur & $J F 724458$ & JF725631 & 31 \\
\hline DT104 & 02-8319 (Clinical isolate) & Institut Pasteur & JF724357 & JF725099 & 24 \\
\hline DT104 & 02-4467 (Clinical isolate) & Institut Pasteur & JF724278 & JF725020 & 23 \\
\hline DT104 & 02-4217 (Clinical isolate) & Institut Pasteur & $J F 724270$ & JF725012 & 20 \\
\hline DT104 & 02-3830 (Clinical isolate) & Institut Pasteur & JF724255 & JF724997 & 22 \\
\hline DT104 & 02-3169 (Clinical isolate) & Institut Pasteur & JF724237 & JF724979 & 21 \\
\hline DT120 & 02-5783 (Clinical isolate) & Institut Pasteur & JF724308 & $J F 725050$ & 21 \\
\hline DT120 & 02-4908 (Clinical isolate) & Institut Pasteur & JF724290 & JF725032 & 34 \\
\hline U302 & 02-3709 (Clinical isolate) & Institut Pasteur & JF724252 & JF724994 & 21 \\
\hline U302 & 02-5064 (Clinical isolate) & Institut Pasteur & JF724292 & JF725034 & 25 \\
\hline DT2 & 81-506 (Veterinary isolate) & Institut Pasteur & JF724622 & JF725354 & 54 \\
\hline DT2 & 01-1639 (Veterinary isolate) & Institut Pasteur & JF724170 & JF724912 & 55 \\
\hline RDNC & 81-748 (Clinical isolate) & Institut Pasteur & JF724624 & JF725356 & 33 \\
\hline RDNC & DK19 (Clinical isolate) & Institut Pasteur & JF724652 & JF725384 & 12 \\
\hline RDNC & 07-4489 (Clinical isolate) & Institut Pasteur & JF724524 & JF725256 & 53 \\
\hline DT1 & 02-0915 (Clinical isolate) & Institut Pasteur & JF724204 & JF724946 & 14 \\
\hline DT40 & 05-2864 (Clinical isolate) & Institut Pasteur & JF724454 & JF725196 & 14 \\
\hline DT1 & $81-481$ (ND) & Institut Pasteur & $J F 724620$ & $J F 725352$ & 11 \\
\hline DT74 & DK24 (Clinical isolate) & Institut Pasteur & JF724648 & JF725380 & 11 \\
\hline DT1 & 1000-7816-1 (Veterinary isolate) & Institut Pasteur & JF724578 & JF725310 & 46 \\
\hline DT186 & 02-1015 (Clinical isolate) & Institut Pasteur & JF724205 & JF724947 & 46 \\
\hline DT12 & 02-2651 (Clinical isolate) & Institut Pasteur & JF724232 & JF724974 & 46 \\
\hline DT42 & 1000-7810-1 (Veterinary isolate) & Institut Pasteur & JF724577 & JF725309 & 46 \\
\hline DT7 & 07-2537 (Clinical isolate) & Institut Pasteur & JF724521 & $J F 725253$ & 1 \\
\hline DT193 & 07-7741 (Clinical isolate) & Institut Pasteur & JF724531 & $J F 725263$ & 1 \\
\hline U311 & 07-8113 (Clinical isolate) & Institut Pasteur & $J F 724532$ & JF725264 & 1 \\
\hline DT41 & 07-5354 (Clinical isolate) & Institut Pasteur & JF724527 & $J F 725259$ & 1 \\
\hline
\end{tabular}

CRISPR type was not determined as the whole genome sequence is not available for these strains

${ }^{*}$ CRISPOL type was determined by Fabre et al. [5]

Interestingly, a strains of phage type DT120 (M162000) has identical spacers and CRISPR/CRISPOL type (9510/1428) as another strain belongs to phage type DT7a (MS120840). Moreover, some strains belong to phage types DT120 (S/20160374 and S/20160407), DT20a (MS150110), DT193 (MS150007) and untypable strain (MS150097) have identical spacers and therefore share the same CRISPR/ CRISPOL type (322/1). Different strains belong to phage type DT193 have different spacers and CRISPR/CRISPOL type; MS150007 (322/1) and MS150252 (317/2).

\section{CRISPR/CRISPOL type among phage types DT3, DT12 and DT193a}

Some strains of phage types DT12 (DT12) and DT3 (S81-482) have identical spacers and identical CRISPR/ CRISPOL type; 5268/19. Moreover, a strain belongs to DT12 (S02-2651) has identical CRISPR/CRISPOL type, 774/46, as a strain belongs to phage type DT193a (MS120454) (Table 2).

\section{CRISPR/CRISPOL type among phage types DT135, DT191a and RDNC}

Identical spacer sequences and CRISPR/CRISPOL type (91/4) were detected in different phage types of $S$. Typhimurium including DT135 (MS150112 and MS150180), DT191a (DT19a) and strains that react with phages but do not confirm to recognized pattern (RDNC) (MS150102 and MS150230). Furthermore, other strains belong to phage type DT135 show different spacers and subsequently different CRISPR/CRISPOL type; 5753/396 in DT135 and 3247/66 in MS150100 (Table 2). 


\section{CRISPOL assay confirms the no relation among phage type and CRISPRs}

CRISPOL assay developed by Fabre et al. [5] when carried out on representative phage types of $S$. Typhimurium it reveals that there is no relation among the phage type and the CRISPOL type as strains belong to the same phage type have different CRISPOL type as seen in DT104 strains (Table 3). On the other hand, different phage types including DT7, DT193, U311, DT41 showed identical CRISPOL type as '1' (Table 3).

\section{Discussion}

Salmonella Typhimurium is the most dominant Salmonella serovar around the world and has been associated with foodborne outbreaks in both developing and highincome countries $[1,11]$ and infection can result in bacteraemia and invasive disease [12, 13]. Epidemiological characterization of $S$. Typhimurium is therefore very crucial for the surveillance and outbreak investigation.

Phage typing system [3] has been a very useful phenotypical, definitive method for epidemiological characterization of $S$. Typhimurium and identification of the source of infection [14-17]. Although it has been suggested that the high throughput CRISPR typing and subtyping have the potential to replace traditional phage typing [5] this study demonstrates that It is impossible for CRISPR typing and CRISPOl assay to replace phage typing for epidemiological characterization of $S$. Typhimurium as there is no correlation between the phage type and the CRISPR/ CRISPOL type.

Interestingly, $S$. Typhimurium DT8 strains associated with the foodborne outbreak in the summer of 2013 in the States of Jersey [10] showed identical CRISPR/ CRISPOL type however, the same CRISPR/CRISPOL type were reported in other DT8 strains that do not belong to the outbreak as confirmed by WGS [10]. Detection of identical spacers among outbreak associated and non-outbreak associated DT8 strains reveals the limitation of CRISPR typing and subtyping in investigation of outbreaks.

The MDR DT104 strain of S. Typhimurium has been associated with foodborne outbreaks all over the world and phage typing was very successful in epidemiological characterization of the outbreak and identification of the source [18-20] however in this study strains belong to DT104 showed different spacers and subsequently different CRISPR/CRISPOL type therefore CRISPR typing and CRISPOL assay cannot be used in public health laboratories to determine the epidemiological relation among $S$. Typhimurium isolates.

The presence of CRISPR/CRISPOL type within the same phage type and the presence of identical spacers among different phage types of $S$. Typhimurium confirms the limitations of CRISPR typing and subtyping for the epidemiological surveillance and outbreak investigation of $S$. Typhimurium.

There is no doubt that rapid WGS will shape the future of diagnostic microbiology as it has the potential to replace the routine typing and subtyping methods including Anderson phage typing system for the surveillance of outbreaks caused by different Salmonella serovars in real-time [10, 21, 22]. However, in the meantime, traditional phage typing scheme of $S$. Typhimurium remains the gold standard method for subtyping of $S$. Typhimurium for laboratory surveillance and outbreak investigation despite its technical limitations. Furthermore, it represents an ideal model for studying the complex dynamics of phage-host interaction [8].

In conclusion, high throughput CRISPR/CRISPOL typing might be useful for the discrimination among different Salmonella serovars however it is not useful for the epidemiological surveillance and outbreak investigation of $S$. Typhimurium and phage typing, until it is replaced by WGS, is still the gold standard method for epidemiological surveillance of $S$. Typhimurium.

\section{Limitations}

More outbreaks of $S$. Typhimurium caused by phage types other than DT8 can be included to confirm the unsuitability of CRISPR typing in epidemiological surveillance and outbreak investigation of $S$. Typhimurium.

\section{Additional file}

Additional file 1: Table S1. CRISPR and CRISPOL types of outbreak and non-outbreak associated DT8 strains of S. Typhimurium. Identical CRISPR and CRISPOL types were detected among outbreak and non-outbreak strains.

\section{Abbreviations}

CRISPR: clustered regular interspaced short palindromic repeats; DT: phage type; MDR: multidrug resistant; NSSLRL: National Salmonella Shigella Listeria Reference Laboratory; NTS: nontyphoidal salmonella; PE: paired end; PCR: polymerase chain reaction; RDNC: strains that react with phages but do not confirm to recognized pattern; SSSCDRL: Scottish Salmonella, Shigella and Clostridium difficile Reference Laboratory; S. Typhimurium: Salmonella Typhimurium; WGS: whole genome sequencing.

\section{Acknowledgements}

Not applicable.

Competing interests

The author no competing interests.

Availability of data and materials

Raw sequence data of control phage types of S. Typhimurium will be publically available via ENA under study Accession No.: PRJEB18673 (http://www. ebi.ac.uk/ena/data/view/PRJEB18673) and also available via Enterobase (https://enterobase.warwick.ac.uk/). All sequencing data is available on request. 


\section{Consent to publish \\ Not applicable.}

\section{Ethical approval and consent}

Not applicable.

\section{Funding}

Not applicable.

\section{Publisher's Note}

Springer Nature remains neutral with regard to jurisdictional claims in published maps and institutional affiliations.

Received: 13 July 2017 Accepted: 26 October 2017

Published online: 07 November 2017

\section{References}

1. Majowicz SE, Musto J, Scallan E, Angulo FJ, Kirk M, O'Brien SJ, Jones TF, Fazil A, Hoekstra RM. International collaboration on enteric disease "burden of illness" S: the global burden of nontyphoidal Salmonella gastroenteritis. Clin Infect Dis. 2010;50(6):882-9.

2. Okoro CK, Kingsley RA, Connor TR, Harris SR, Parry CM, Al-Mashhadani MN, Kariuki S, Msefula CL, Gordon MA, de Pinna E, et al. Intracontinental spread of human invasive Salmonella typhimurium pathovariants in subSaharan Africa. Nat Genet. 2012;44(11):1215-21.

3. Anderson ES, Ward LR, Saxe MJ, de Sa JD. Bacteriophage-typing designations of Salmonella typhimurium. J Hyg (Lond). 1977;78(2):297-300.

4. Baggesen DL, Sorensen G, Nielsen EM, Wegener HC. Phage typing of Salmonella typhimurium - is it still a useful tool for surveillance and outbreak investigation? Euro surveillance: bulletin European sur les maladies transmissibles. Eur Commun Dis Bull. 2010;15(4):19471.

5. Fabre L, Zhang J, Guigon G, Le Hello S, Guibert V, Accou-Demartin M, de Romans S, Lim C, Roux C, Passet $\mathrm{V}$, et al. CRISPR typing and subtyping for improved laboratory surveillance of Salmonella infections. PLoS ONE. 2012;7(5):e36995.

6. Shariat N, Dudley EG. CRISPRs: molecular signatures used for pathogen subtyping. Appl Environ Microbiol. 2014;80(2):430-9.

7. Jansen R, Embden JD, Gaastra W, Schouls LM. Identification of genes that are associated with DNA repeats in prokaryotes. Mol Microbiol. 2002:43(6):1565-75

8. Mohammed M, Cormican M. Whole genome sequencing provides possible explanations for the difference in phage susceptibility among two Salmonella typhimurium phage types (DT8 and DT30) associated with a single foodborne outbreak. BMC Res Notes. 2015:8:728.

9. Zerbino DR, Birney E. Velvet: algorithms for de novo short read assembly using de Bruijn graphs. Genome Res. 2008;18(5):821-9.

10. Ashton PM, Nair S, Peters TM, Bale JA, Powell DG, Painset A, Tewolde R, Schaefer U, Jenkins C, Dallman TJ, et al. Identification of Salmonella for public health surveillance using whole genome sequencing. PeerJ. 2016;4:e1752

11. Galanis E, Lo Fo Wong DM, Patrick ME, Binsztein N, Cieslik A, Chalermchikit T, Aidara-Kane A, Ellis A, Angulo FJ, Wegener HC, et al. Web-based surveillance and global Salmonella distribution, 2000-2002. Emerg Infect Dis. 2006;12(3):381-8.
12. Ao TT, Feasey NA, Gordon MA, Keddy KH, Angulo FJ, Crump JA. Global burden of invasive nontyphoidal Salmonella disease, 2010. Emerg Infect Dis. 2015;21(6):941.

13. Tapia MD, Tennant SM, Bornstein K, Onwuchekwa U, Tamboura B, Maiga A, Sylla MB, Sissoko S, Kourouma N, Toure A, et al. Invasive nontyphoidal Salmonella infections among children in Mali, 2002-2014: microbiological and epidemiologic features guide vaccine development. Clin Infect Dis. 2015;61(Suppl 4):S332-8.

14. Fielding JE, Snell P, Milazzo A, Del Fabbro L, Raupach J. An outbreak of Salmonella typhimurium phage type 4 linked to cold set cheesecake. Commun Dis Intell Q Rep. 2003;27(4):513-4

15. Tribe IG, Tsimogiannis H, Mmolawa P, Davos D. An outbreak of Salmonella typhimurium phage type 29 linked to a noodle restaurant in South Australia. Commun Dis Intell Q Rep. 2001;25(2):72.

16. Moffatt CR, Combs BG, Mwanri L, Holland R, Delroy B, Cameron S, Givney RC. An outbreak of Salmonella typhimurium phage type 64 gastroenteritis linked to catered luncheons in Adelaide, South Australia, June 2005. Commun Dis Intell Q Rep. 2006:30(4):443-8.

17. Boxall NS, Adak GK. E DEP, Gillespie IA: a Salmonella typhimurium phage type (PT) U320 outbreak in England, 2008: continuation of a trend involving ready-to-eat products. Epidemiol Infect. 2011;139(12):1936-44.

18. Molbak K, Baggesen DL, Aarestrup FM, Ebbesen JM, Engberg J, Frydendahl K, Gerner-Smidt P, Petersen AM, Wegener HC. An outbreak of multidrug-resistant, quinolone-resistant Salmonella enterica serotype typhimurium DT104. N Engl J Med. 1999;341(19):1420-5.

19. Cody SH, Abbott SL, Marfin AA, Schulz B, Wagner P, Robbins K, MohleBoetani JC, Vugia DJ. Two outbreaks of multidrug-resistant Salmonella serotype typhimurium DT104 infections linked to raw-milk cheese in northern California. JAMA. 1999:281(19):1805-10.

20. Dechet AM, Scallan E, Gensheimer K, Hoekstra R, Gunderman-King J, Lockett J, Wrigley D, Chege W, Sobel J, Multistate Working G. Outbreak of multidrug-resistant Salmonella enterica serotype typhimurium definitive type 104 infection linked to commercial ground beef, northeastern United States, 2003-2004. Clin Infect Dis. 2006;42(6):747-52.

21. Mohammed M, Delappe N, O'Connor J, McKeown P, Garvey P, Cormican $M$. Whole genome sequencing provides an unambiguous link between Salmonella Dublin outbreak strain and a historical isolate. Epidemiol Infect 2016;144(3):576-81.

22. Wuyts V, Denayer S, Roosens NH, Mattheus W, Bertrand S, Marchal K, Dierick K, De Keersmaecker SC. Whole genome sequence analysis of Salmonella enteritidis PT4 outbreaks from a National Reference Laboratory's Viewpoint. PLoS curr 2015. https://doi.org/10.1371/currents.outbreaks. aa5372d90826e6cb0136ff66bb7a62fc

\section{Submit your next manuscript to BioMed Central and we will help you at every step:}

- We accept pre-submission inquiries

- Our selector tool helps you to find the most relevant journal

- We provide round the clock customer support

- Convenient online submission

- Thorough peer review

- Inclusion in PubMed and all major indexing services

- Maximum visibility for your research

Submit your manuscript at www.biomedcentral com/submit 